\title{
Research and analysis on defect detection of semi-conductive layer of high voltage cable
}

\author{
Sanwei Liu ${ }^{1}$, Jun Zhang ${ }^{1}$, Yi Xie ${ }^{1}$, Jianjia Duan ${ }^{1}$, Fuyong Huang ${ }^{1}$, Xiaoli Duan ${ }^{1}$, Zeyu Zeng ${ }^{1}$ \\ ${ }^{1}$ State grid Hunan electric power co, LTD. Electric power research institute, Changsha, Hunan, 410000, China
}

\begin{abstract}
As a component of the Internet of things, high-voltage cables are the power supply infrastructure for the modern development of cities. The operation experience shows that the high-voltage cable has been broken down many times due to the defective operation. At present, due to the limitation of detection technology, the research on detection and identification of defects in high-voltage cables is progressing slowly. Therefore, a new DR technology based on X-ray digital imaging is proposed in this paper to realize real-time detection of defects in the semi-conductive buffer layer of high-voltage cables, and intelligent detection of DR images of high-voltage cables by using image depth processing technology to realize intelligent identification of defects in the buffer layer of power cables. The results show that using the new DR technique proposed in this paper, the accurate and intuitive DR image of high-voltage cable can be obtained quickly, and the intelligent identification of defects can be realized.
\end{abstract}

\section{Introduction}

High voltage cable is an important infrastructure equipment for building the Internet of things. Operation experience shows that under the influence of production process, transportation and storage, distribution defects will occur in the semi conducting buffer layer of high-voltage cable, which is easy to cause breakdown of cable body under random insulation during operation, and the probability of breakdown increases significantly with the increase of operation time, Over the past 20 years, the defects in the high-voltage cable industry have shown multiple natures, with problems such as factory distribution, unclear defect mechanisms, imperfect defect detection methods, and difficulty in implementing status assessments[1]. Before that, the State Grid Corporation and the South Grid Corporation decided that "the existing common high-voltage cable online monitoring means are difficult to obtain such defects effectively[2].

As an effective non-destructive testing method, X-ray Digital Radiography (DR) was first used in the medical, aerospace and other industries, and has gradually been widely used in materials, machinery and other industrial fields[3-4]. In order to meet the demand of power Internet of things construction, this technology has also been applied in the field of state perception of power transmission and transformation equipment. Typical application scenarios include transmission line tension line clamping and compression quality test and structure test of substation GIS equipment[5].

Different from tension clamps and GIS equipment, due to the special structure of high-voltage cables, there is still no effective method to detect and identify the defects of the semi-conductive buffer layer of high-voltage cables. Therefore, this paper proposes a new Image Depth Intelligent processing-digital Radiography (DR) technology to realize Intelligent recognition of high-voltage cable X-ray Digital Image Processing and power cable buffer layer defects. By turning passivity into initiative, the insulation breakdown accident in the operation of high-voltage cable can be forewarned in advance, and secondary disasters such as cable fire and loss of important section of power grid can be avoided.

\section{DR detection technology}

X-rays of different energies have different abilities to penetrate the material being examined. Theoretical and experimental studies show that when narrow beams and single-energy $\mathrm{x}$-rays pass through a layer of material of uniform thickness, their intensity decays exponentially, and the incident intensity decays with the increase of the thickness of the penetrating object, that is:

$$
I=I_{0} e^{-\mu \rho x}
$$

The plate detector is used to receive the X-ray that penetrates the detected workpiece, and then the crystal circuit inside the plate detector converts it into the current signal according to the X-ray dose intensity, and finally presents it in the form of digital image on the terminal computer[6].

\section{Defect generation mechanism}

A large number of cable disintegration tests with buffer layer defects show that there are a large number of white powdery substances in the cable buffer layer with water resistance structure, aluminum sheath and insulation shielding layer. Under the action of operating voltage for

*Corresponding author's e-mail: 604208086@qq.com 
a long time, the defect of the buffer layer gradually develops, ablates and runs through the semi-conductive layer of the cable, finally leading to the breakdown of the main insulation of the cable. According to the infrared spectrum, the white powdery substance contains sodium, aluminum and other metal elements.

The main component of the water-blocking powder in the water-belt buffer layer of cable semi-conducting resistance is low-crosslinked polyacrylate sodium salt, which has strong water absorption. When the cable is poorly controlled due to production, storage, transportation, construction and other links, the semi-conductive resistance water buffer layer will absorb water and be affected by moisture, forming free $\mathrm{OH}$ - and $\mathrm{Na}+$ ions. When the $\mathrm{OH}$ - ions absorb $\mathrm{CO} 2$ in the air, they will form sodium carbonate $(\mathrm{Na} 2 \mathrm{CO} 3)$ or sodium bicarbonate (NaHCO3) :

Small amount of $\mathrm{CO} 2$ :

$$
\begin{gathered}
\mathrm{CO}_{2}+2 \mathrm{OH}^{-}=\mathrm{CO}_{3}{ }^{2-}+\mathrm{H}_{2} \mathrm{O} \\
\mathrm{CO}_{3}{ }^{2-}+2 \mathrm{Na}^{+}=\mathrm{Na}_{2} \mathrm{CO}_{3}
\end{gathered}
$$

A glut of $\mathrm{CO} 2$ :

$$
\mathrm{CO}^{2}+\mathrm{OH}^{-}=\mathrm{HCO}_{3}^{-}
$$$$
\mathrm{HCO}_{3}{ }^{-}+\mathrm{Na}^{+}=\mathrm{NaHCO}_{3}
$$

Sodium carbonate $(\mathrm{Na} 2 \mathrm{CO} 3)$ or sodium bicarbonate (NaHCO3) are white powder crystals. Therefore, the sodium element in the white powder exists in the form of sodium bicarbonate or sodium bicarbonate.In addition, the water-blocking powder forms a free $\mathrm{OH}$ - ion, which contacts with the buffer layer on the inside of the trough of the aluminum sheath, and a chemical reaction occurs between the $\mathrm{OH}$ - ion and the aluminum, resulting in AlO2-, an aluminum-biased acid radical:

$$
2 \mathrm{Al}+2 \mathrm{OH}^{-}+2 \mathrm{H}_{2} \mathrm{O}=2 \mathrm{AlO}_{2}^{-}+3 \mathrm{H}_{2}
$$

$\mathrm{AlO} 2-$ and $\mathrm{Na}+$ ions in the water blocking powder form $\mathrm{NaAlO} 2$, and the reaction produces $\mathrm{NaAlO} 2$, which is easily soluble in water, and absorbs $\mathrm{CO} 2$ in the air and reacts to form $\mathrm{Al}(\mathrm{OH}) 3:$ :

Small amount of $\mathrm{CO} 2$ :

$$
\mathrm{AlO}_{2}{ }^{-}+\mathrm{CO}_{2}+2 \mathrm{H}_{2} \mathrm{O}=\mathrm{Al}(\mathrm{OH})_{3}+\mathrm{HCO}_{3}
$$

A glut of $\mathrm{CO} 2$ :

$$
2 \mathrm{AlO}_{2}^{-}+\mathrm{CO}_{2}+3 \mathrm{H}_{2} \mathrm{O}=2 \mathrm{Al}(\mathrm{OH})_{3}+\mathrm{CO}_{3}^{2-}
$$

Finally, the generated aluminum hydroxide $\mathrm{Al}(\mathrm{OH}) 3$ is unstable in nature and is easy to decompose into aluminum oxide $\mathrm{A} 12 \mathrm{O} 3$ :

$$
2 \mathrm{Al}(\mathrm{OH})_{3}=\mathrm{Al}_{2} \mathrm{O}_{3}+3 \mathrm{H}_{2} \mathrm{O}
$$

Therefore, the main components of the white powder are sodium carbonate $(\mathrm{Na} 2 \mathrm{CO} 3)$, sodium bicarbonate (NaHCO3) and alumina (Al2O3) crystals.

Most of the white powder at the defects was generated at the trough where the aluminum sheath had the closest contact with the buffer layer. The reason was that the sodium aluminate $\mathrm{NaAlO} 2$ produced by the above reaction was easily soluble in water, and the sodium aluminate ions would migrate around the contact point between the aluminum sheath and the buffer layer and inside the buffer layer under the effect of concentration gradient. On the other hand, the solution will produce electrophoresis phenomenon under the electric field, and the particles with high permittivity will move in the direction of the high electric field, which will cause the sodium aluminate here to accumulate towards the insulation shield of the cable core.

\section{$4 \quad$ Field application}

\subsection{Field briefing}

The distribution diagram of cable line is shown in figure 1. Line A, line B and line $\mathrm{M}$ of $110 \mathrm{kV}$ are derived from the tea garden at intervals of 504 through the cable, and laid along the "220 kV X to $220 \mathrm{kV} \mathrm{Y}$ cable tunnel (about $2400 \mathrm{~m}$ ). The total length of the cable is about $3,100 \mathrm{~m}$, and it is divided into four sections, including two tunnel segment joints and one directly buried segment joint. At section III of the tunnel, it is laid with lines $\mathrm{C}$ and $\mathrm{D}$ and $\mathrm{E}$ of $110 \mathrm{kV}$.

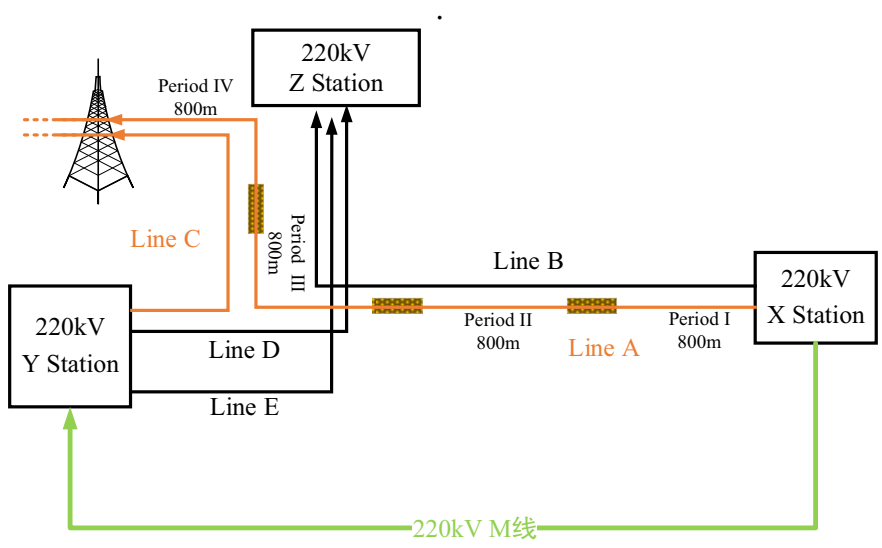

Fig.1 Cable tunnel diagram

The circuit diagram of line $\mathrm{A}$ of $110 \mathrm{kV}$ is shown in figure 6. The detection scheme takes two joints as A section and changes from $\mathrm{X}$ to $\mathrm{Y}$, as shown in figure 1 . There are altogether 4 sections, which can be labeled as I, II, III, and IV sections, each of which is about $800 \mathrm{~m}$ long. 8 points are detected in each section, and the interval between each point is $100 \mathrm{~m}$, marked as $1,2,3$.. 8 PM. The detection points of $\mathrm{A}, \mathrm{B}$ and $\mathrm{C}$ are set in this way.

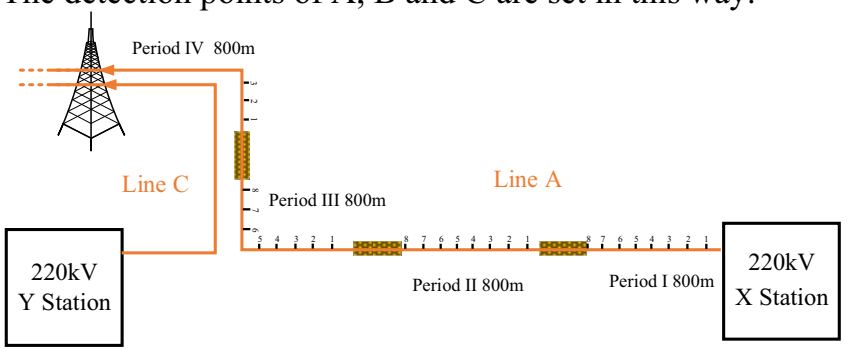

Fig.2 Check point layout

\subsection{Test results and analysis}

$\mathrm{A}, \mathrm{B}$ and $\mathrm{C}$ of $110 \mathrm{kV}$ tea chrysanthemum steel line are cables made in lingnan, guangzhou. A total of 64 tests have been completed in sections I, II, III and IV, and 21 defect points and 43 normal points have been detected. 
In this paper, full convolutional neural network FCN is used for defect identification. The DR detection image of normal cable is shown in figure 3 below:
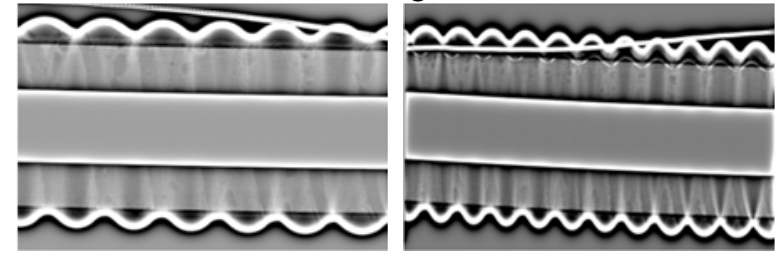

(a) horizontal X-ray image (b) vertical X-ray image

Fig.3 Line A normal cable DR detection image

Using the DR detection technology proposed in this paper, the whole line of line A was tested by DR, and the defective cable segments were found: A phase I, II, III, B phase I, II, III, IV, C phase I, II, III, IV. The DR detection image of a typical defective cable is shown in figure 4 .
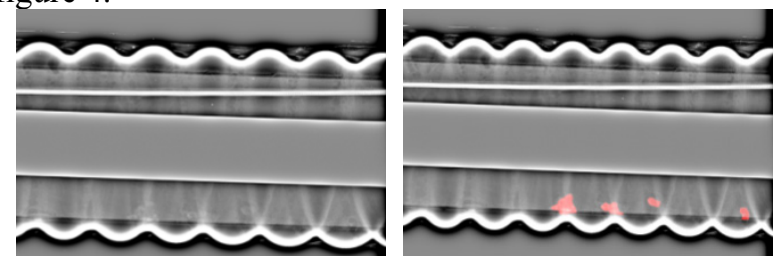

(a) original drawing $\quad$ (b) defect identification drawing (1) A-phase defective cable

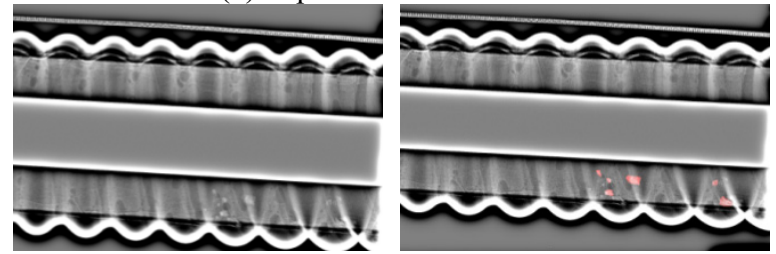

(a) original drawing (b) defect identification drawing

(2) B-phase defective cable

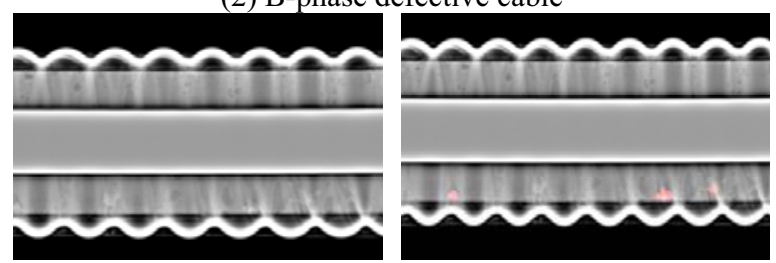

(a) original drawing (b) defect identification drawing (3) C-phase defective cable

Fig.4 Line A defect cable DR detection image

Characteristic diagnosis and analysis are carried out for the DR image data set detected in the field. The DR image of the cable buffer layer has the following characteristics:

(1) cable buffer layer defects during imaging, if the defects are on the same section as the incident X-ray and cable core, the cable defects will be covered by the cable core. Therefore, during X-ray detection, at least two images should be taken, and the angles of the two images should cover $360^{\circ}$ to ensure that the defects can be photographed.

(2) most of the defects occurred at the trough where the aluminum sheath and buffer layer had the closest contact, and the test results were consistent with the theoretical analysis in 1.2.

(3) the defect of the cable buffer layer has a significant position feature: when shooting in the horizontal direction, the defect is basically located at the bottom of the image, and the corresponding actual position is at the bottom of the cable; When shooting vertically, the defect is basically located at the top of the image, and the corresponding actual position is the cable near the inside of the wall. Therefore, from the image analysis, the cable buffer layer defect is basically located in the cable bottom inner region.

\section{Conclusion}

Aiming at the problem of defect detection and recognition in the buffer layer of high voltage cable, the validity of the DR detection method proposed in this paper is proved by the spot defect detection, and the rule of the defect detection in the buffer layer is summarized.

\section{References}

1. Duarte MF, Davenport MA, Takhar D, Sun T, Kelly KF, Baranuik RG. Signal-pixel imaging via compressive sampling [J]. IEEE Signal Processing Magazine, 2008, 25(2): 83-91.

2. [Gan H, Cheng Z, Yang S, et al. Circulant and toeplitz chaotic matrices in compressed sensing [J]. Journal of Computational Information Systems, 2015, 11(4): 1231-1238.

3. Gan H, Cheng Z, Yang S, et al. Circulant and toeplitz chaotic matrices in compressed sensing [J]. Journal of Computational Information Systems, 2015, 11(4): 1231-1238.

4. Duarte MF, Davenport MA, Takhar D, Sun T, Kelly KF, Baranuik RG. Signal-pixel imaging via compressive sampling $[\mathrm{J}]$. IEEE Signal Processing Magazine, 2008, 25(2): 83-91.

5. Sun Y, Wang X, Tang X . Deep Learning Face Representation from Predicting 10,000 Classes[C]// 2014 IEEE Conference on Computer Vision and Pattern Recognition (CVPR). IEEE Computer Society, 2014.

6. Srinivasan V, Sankar A R, Balasubramanian V N. ADINE: An Adaptive Momentum Method for Stochastic Gradient Descent[J]. 2017. 